\title{
CLUSTERS, CORRELATIONS AND TRANSVERSE MOMENTA
}

\author{
F. HAYOT *, F.S. HENYEY and M. LEBELLAC ** \\ Department of Physics, University of Michigan, Ann Arbor, Michigan 48104 ***
}

Received 4 June 1974

\begin{abstract}
We discuss the short range part of two-particle correlations as it results from the phase space available in cluster decay. In such an approach, certain variables emerge which should be useful to organize the data and extract interesting information. We are in particular conccrned with tests of large transverse motion of clusters.
\end{abstract}

\section{Introduction}

The observed short range rapidity correlations between two particles have been often interpreted in terms of clustering [1-5]. Aside from a rough comparison with the shape of the correlation, this interpretation has been without a test. The magnitude of the correlation is fit by adjusting the cluster size.

Recently the idea of clustering has also been used to describe the single particle inclusive transverse momentum distribution. Again the distribution of cluster masses is adjusted to fit the transverse momentum dependence of the inclusive cross section. [6]. In fact there is so much freedom that the additional assumption of no transverse cluster motion can be made.

Thus one has to study more detailed data if one wishes to test the clustering hypothesis. The ultimate goal of course is to be able to tell whether clustering is merely a convenient language, or whether clusters are actually produced. It therefore becomes of interest to determine the cluster parameters further and use for this purpose the full dependence of the two-particle correlation on the four kinematical variables, and not only its dependence on rapidity differences.

One of the most important points we wish to address ourselves to in this paper is the question of whether clusters can have a large transverse momentum. In fact the idea of clustering has been used to circumvent the difficulties of two standard models of multiparticle production with respect to elastic scattering [7-9]. It is known that the independent emission model (IEM) and the multiperipheral model

\footnotetext{
* Permanent address: Service de Physique Théorique, C.E.N. Saclay.

** Permanent address: Faculté des Sciences, Université de Nice.

*** Work supported by the US Atomic Energy Commission.
} 
(MPM) give wrong predictions for the overlap function, if these models are used to describe the production of particles. This point will be discussed in more detail in sect. 2.

In sect. 3 we study two-particle correlations as they result from cluster phasespace. We point out some characteristic features, related both to a reasonable choice of variables with which to organize the data, and ways to study cluster transverse motion.

The general framework which we adopt is the following: we assume that in a typical high energy collision several clusters of particles are produced via an unspecified dynamical mechanism. We assume the existence of a central region in rapidity, where only rapidity differences between final particles matter (short range order). In this region, clusters are produced uniformly in rapidity and their production can be specified as a function of the cluster mass $M$ and transverse momentum $K_{\perp}$. The clusters decay independently of production, except that, of course, the decay depends on the cluster mass. In particular, we assume that clusters decay isotropically in their rest frame.

As sect. 3 is unfortunately rather technical, we finally summarize in sect. 4 those aspects and results of our work which can be of interest to experimentalists.

\section{Elastic scattering and cluster transverse momentum}

In a cluster model, the transverse momentum $\boldsymbol{p}_{1}$ of an observed particle is the combination of the cluster transverse momentum $K_{\perp}$ and of the particle transverse momen. $\operatorname{tum} q_{\perp}$ in the cluster rest frame. In order to get a feeling about the order of magnitude involved, we first derive a general formula which relates $\left\langle p_{\perp}^{2}\right\rangle$ to $\left\langle K_{\perp}^{2}\right\rangle$ and $\left\langle q_{\perp}^{2}\right\rangle$. This formula will allow us to define what is a typically small value of $\left\langle K_{\perp}^{2}\right\rangle$ and what is on the contrary a typically large value of $\left\langle K_{1}^{2}\right\rangle$. Interestingly encugh, these two cases will be associated with the independent emission model and the multiperipheral model respectively.

Consider a cluster that is boosted from rest to a momentum $K_{\perp}$ along the $x$-direction (the $z$-axis being, as usual, the axis defined by the incident particles). If $\eta$ is the cluster transverse rapidity and $M_{\perp}=\sqrt{M^{2}+K_{\perp}^{2}}$ the cluster transverse mass:

$$
M_{\perp}=M \cosh \eta, \quad K_{\perp}=M \sinh \eta,
$$

the particle momentum is given after the boost by:

$$
\begin{aligned}
& p_{x}=q_{x} \cosh \eta+E \sinh \eta, \\
& p_{y}=q_{y} .
\end{aligned}
$$

Isotropy of the cluster decay gives

$$
\left\langle q_{x}\right\rangle=0, \quad\left\langle q_{x}^{2}\right\rangle=\left\langle q_{y}^{2}\right\rangle=\frac{1}{2}\left\langle q_{\perp}^{2}\right\rangle=\frac{1}{3}\left\langle E^{2}-\mu^{2}\right\rangle .
$$


Averaging first over the decay distribution, and then over cluster production (which is possible since both processes are independent) we get:

$$
\left\langle p_{\perp}^{2}\right\rangle=\left\langle q_{\perp}^{2}\right\rangle\left(1+2\left\langle K_{\perp}^{2} / M^{2}\right\rangle\right)+\mu^{2}\left\langle K_{\perp}^{2} / M^{2}\right\rangle
$$

where $\mu$ is the pion mass. The last term in (2) can be usually neglected; it would become of importance only if the particles were almost at rest in the cluster rest frame, which would have been easily discovered experimentally.

As a rough estimate of (2), we can choose *

$$
\left\langle q_{L}^{2}\right\rangle \sim\langle E\rangle^{2} \sim \frac{\langle M\rangle^{2}}{\langle n\rangle^{2}},
$$

where $\langle n\rangle$ is the average number of particles within a cluster. Experimentally $\left\langle p_{\perp}^{2}\right\rangle \sim 0.2(\mathrm{GeV})^{2}$ while various estimates indicate that $\langle n\rangle \sim 4$ is a fair guess $[2,3$, 5]. We then obtain in the two cases, $\left\langle K_{\perp}^{2}\right\rangle=0$ and $\left\langle K_{\perp}^{2}\right\rangle=1 \mathrm{GeV}^{2}$ :

(i) $\left\langle K_{\perp}^{2}\right\rangle=0,\left\langle q_{\perp}^{2}\right\rangle=0.2 \mathrm{GeV}^{2}, M \simeq 1.8 \mathrm{GeV}$,

(ii) $\left\langle K_{\perp}^{2}\right\rangle-1 \mathrm{GcV}^{2},\left\langle q_{\perp}^{2}\right\rangle=0.075 \mathrm{GeV}^{2}, M \simeq 1.1 \mathrm{GeV}$.

It is clear from (2) that a cluster transverse momentum is large whenever it is of the same order as the cluster mass. We wish now to show that cases (i) and (ii) can be associated with IEM and MPM respectively.

In IEM, assuming that the leading clusters (or particles) carry off most of the available angular momentum, it is easy to show, either by a direct calculation, or from the impact parameter analysis of ref. [7], that the overlap function is proportional to

$$
\exp \left(\frac{t}{2\left\langle K^{2}\right\rangle}\right)
$$

From data on elastic scattering, we know that the argument in the exponential is $\sim 6 t$, so that

$$
\left\langle K_{1}^{2}\right\rangle \sim \frac{1}{12} \sim 0.08 \mathrm{GeV}^{2},
$$

which must be considered as a small number **: indeed we can see from (2) that with such a value of $\left\langle K_{\perp}^{2}\right\rangle$, and with $M^{2} \sim 3$ nost of the particle transverse motion is accounted for by the motion within the cluster.

On the other hand, MPM correspond to a random walk in impact parameter space. In simple versions this leads to a proportionality, as a function of the incident energy, between the elastic slope and the number of clusters minus one, which is in

* A more accurate calculation will be presented in sect. 3, where we use a specific model of cluster decay.

** As is well-known, inclusion of phases would lead to a larger value of $\left\langle K_{\perp}^{2}\right\rangle$, and our estimate is a lower bound. 
contradiction with the data. In order to remedy this, Caneschi [10] has suggested that the first and last step in the random walk are several times as large as the central steps. This adds a constant $A_{1}$ to the elastic slope $B$ which is given by:

$$
B \simeq A_{1}+\langle N\rangle /\left(\langle n\rangle\left\langle K_{\perp}^{2}\right\rangle\right)
$$

where $\langle N\rangle$ is the average number of particles produced in the collision. Since the multiplicity and the slope are logarithmic in MPM, we can identify the coefficients of $\log s$ in (4) with that in the usual expression

$$
B=A_{1}^{\prime}+2 \alpha_{\mathrm{p}}^{\prime} \log s
$$

where $\alpha_{\mathrm{p}}^{\prime} \simeq 0.25(\mathrm{GeV})^{-2}$. The coefficient of $\log s$ in $\langle N\rangle$ is of the order of 2.5 , so that we get by comparison of (4) and (5):

$$
\left\langle K_{\perp}^{2}\right\rangle \sim \frac{5}{\langle n\rangle} \sim 1.2 \mathrm{GeV}^{2} .
$$

This value is again a lower estimate, but we see that in any case MPM is to be associated with typically large values of $\left\langle K_{\perp}^{2}\right\rangle$.

To summarize: We can say that the cluster transverse momentum is small (large) whenever $2\left(K^{2} / M^{2}\right)$ in (2) is smaller (larger) than one. Provided our order of magnitude arguments are not spoiled by subleties about phases etc...., we can associate small $\left\langle K_{\perp}^{2}\right\rangle$ with IEM, large $\left\langle K_{\perp}^{2}\right\rangle$ with MPM.

\section{One- and two-particle distributions in cluster models}

Let us state again what we exactly mean by cluster models. Our first assumption is that clusters are produced independently. The implication of azimuthal or long range correlations between clusters will be treated later. The short range part of the correlation between two particles is then obtained when both particles originate from the same cluster.

We next assume that phase-space controls the properties of cluster decay (more generally we could assume a smoothly varying matrix element leading to isotropic decay). If the description in terms of cluster production and decay is not merely a convenient language, but corresponds to a true physical mechanism, our assumption means that the dynamics of the multiparticle process is buried in the cluster formation, whereas the two-particle correlation is determined from the kinematics of the decay alone.

\subsection{General discussion}

Let us concentrate on one cluster. The probability that a cluster with mass $M$ decays into $n$ particles is proportional to

$$
\Gamma_{n}\left(K^{2}\right)=\int \frac{\mathrm{d}^{3} p_{1}}{E_{1}} \cdots \frac{\mathrm{d}^{3} p_{n}}{E_{n}} \delta^{(4)}\left(K-\sum_{1}^{n} p_{i}\right)
$$


where $K$ is the cluster four-momentum $\left(K^{2}=M^{2}\right)$. The Lorentz invariant one-particle distribution $F$ is then given by:

$$
F\left((K-p)^{2}\right)=\frac{\sum_{n} n g_{n} \Gamma_{n-1}\left((K-p)^{2}\right)}{\sum_{n} g_{n} \Gamma_{n}\left(K^{2}\right)},
$$

where $g_{n}$ is some coupling constant. A common choice is [6]

$$
g_{n}=\frac{g^{n}}{n !}
$$

but we do not want to make this choice here. We remark, for further use, that $F$ is an increasing function of its argument. Similarly the two-particle distribution $\widetilde{F}$ is given by:

$$
\widetilde{F}\left(\left(K-p_{1}-p_{2}\right)^{2}\right)=\frac{\sum_{n} g_{n} n(n-1) \Gamma_{n-2}\left(\left(K-p_{1}-p_{2}\right)^{2}\right)}{\sum_{n} g_{n} \Gamma_{n}\left(K^{2}\right)},
$$

Integration of $F$ with the measure $\mathrm{d}^{3} p / E$ and of $\widetilde{F}$ with the measure $\mathrm{d}^{3} p_{1} \mathrm{~d}^{3} p_{2} / E_{1} E_{2}$ give $\langle n\rangle$ and $\langle n(n-1)\rangle$ respectively. The main point about $(7)$ and $(8)$ is that the one and two particle distributions depend only on the missing mass $m^{2}=(K-p)^{2}$ or $\left(K-p_{1}-p_{2}\right)^{2}$.

The cross section $\rho(p)$ for observing one particle of momentum $p$ for the c.m. frame of the reaction is obtained by integrating ( 7 ) over the cluster mass, rapidity and transverse momentum distribution. Let $f\left(K_{\perp}, M\right)$ be the cross section for producing a cluster of mass $M$ and transverse momentum $K_{\perp}$ normalized as follows:

$$
\int \mathrm{d} Y \mathrm{~d}^{2} K_{\perp} \mathrm{d} M f\left(K_{\perp}, M\right)=\left\langle n_{\mathrm{dl}}\right\rangle,
$$

where $\left\langle n_{\mathrm{cl}}\right\rangle$ is the average number of clusters. Since we are interested in the central region, $f$ is independent of the cluster rapidity $Y$. The missing mass $m^{2}=(K-p)^{2}$ can be written as:

$$
m^{2}=M^{2}+\mu^{2}-2 M_{\perp} \mu_{\perp} \cosh Y+2 K_{\perp} p_{\perp} \cos \Phi,
$$

where $\mu_{\perp}$ is the pion transverse mass, $\Phi$ is the azimuthal angle between $K_{\perp}$ and $p_{\perp}$, and $Y$ is the difference between the pion and cluster rapidities. The one-particle cross section $\rho(p)$ is then given by:

$$
\rho(p)=\int \mathrm{d} M \mathrm{~d} Y \mathrm{~d} K_{\perp} \mathrm{d} \Phi K_{\perp} f\left(K_{\perp}, M\right) F\left(M^{2}+\mu^{2}-2 M_{\perp} \mu_{\perp} \cosh Y+2 K_{\perp} p_{\perp} \cos \Phi\right) .
$$

In order to write the two-particle distribution $\widetilde{C}\left(p_{1}, p_{2}\right)$ we introduce the invariant mass $\tilde{\mu}$ and the transverse mass $\widetilde{\mu}_{\perp}$ of the system formed by the two particles:

$$
\begin{aligned}
& \widetilde{\mu}^{2}=\left(p_{1}+p_{2}\right)^{2}, \\
& \widetilde{\mu}_{\perp}^{2}=\widetilde{\mu}^{2}+\left(p_{1 \perp}+p_{2 \perp}\right)^{2}=\widetilde{\mu}^{2}+\widetilde{p}_{\perp}^{2},
\end{aligned}
$$


the missing mass $m^{2}=\left(K-p_{1}-p_{2}\right)^{2}$ is then expressed as:

$$
m^{2}=M^{2}+\tilde{\mu}^{2}-2 M_{\perp} \tilde{\mu} \cosh Y+2 K_{\perp} \tilde{p}_{\perp} \cos \Phi .
$$

It is then clear that the expression for $\widetilde{C}\left(p_{1}, p_{2}\right)$ is identical to (11) with the substitutions $\mu \rightarrow \widetilde{\mu}, \mu_{\perp} \rightarrow \widetilde{\mu}_{1}, F \rightarrow \widetilde{F}$. We recall that $\widetilde{C}\left(p_{1}, p_{2}\right)$ is that part of the two-particle inclusive cross section where both particles originate from the same cluster. If the clusters are produced independently, $\widetilde{C}\left(p_{1}, p_{2}\right)$ is the usual correlation. We now notice that $\widetilde{C}\left(p_{1}, p_{2}\right)$ depends only on the two variables $\widetilde{\mu}$ and $\widetilde{\mu}_{\perp}$. Thus we can make the following important point: although the correlation in the central region depends a priori on four variables (for example $\left(y_{1}, y_{2}\right), p_{1 \perp}, p_{2 \perp}$, and the azimuthal angle $\varphi$ ), in a model of independent clusters decaying via phase space this correlation depends only on the two combinations $\widetilde{\mu}$ and $\widetilde{\mu}_{\perp}$ formed with these variables:

$$
\begin{aligned}
& \widetilde{\mu}^{2}=2 \mu^{2}+2 \mu_{1 \perp} \mu_{2 \perp} \cosh \left(y_{1}-y_{2}\right)-2 p_{1 \perp} p_{2 \perp} \cos \varphi, \\
& \tilde{\mu}_{\perp}^{2}=\mu_{1 \perp}^{2}+\mu_{2 \perp}^{2}+2 \mu_{1 \perp} \mu_{2 \perp} \cosh \left(y_{1}-y_{2}\right) .
\end{aligned}
$$

Since only $\mu^{2}$ depends on the azimuthal angle $\varphi$, it is very easy to understand qualitatively the $\varphi$ dependence of the correlation. Let us calculate the derivative with respect to $\widetilde{\mu}^{2}$ of the correlation written as a function of $\widetilde{\mu}^{2}$ and $\widetilde{\mu}_{1}^{2}$ :

$$
\begin{aligned}
& \frac{\partial}{\partial \widetilde{\mu}^{2}} \widetilde{C}\left(\tilde{\mu}^{2}, \widetilde{\mu}_{\perp}^{2}\right)=\int \mathrm{d} Y \mathrm{~d} M \mathrm{~d} P_{\perp} \mathrm{d} \Phi K_{\perp} f\left(K_{\perp}, M\right) \\
& \quad \times\left(1-\frac{K_{\perp}}{\widetilde{p}_{\perp}} \cos \Phi\right) \widetilde{F}^{\prime}\left(M^{2}+\widetilde{\mu}^{2}-2 M_{\perp} \widetilde{\mu}_{\perp} \cosh Y+2 K_{\perp} \widetilde{p}_{\perp} \cos \Phi\right) .
\end{aligned}
$$

First it is obvious from (15) that if the clusters have negligible transverse motion, $\widetilde{C}$ is an increasing function of $\widetilde{\mu}^{2}$ since $\widetilde{F}^{\prime} \geqslant 0$ : the two-particle correlation will then be larger at $\varphi=180^{\circ}$ than at $\varphi=0^{\circ}$. On the contrary, when the cluster transverse motion becomes large, $\widetilde{C}$ will finally become a decreasing function of $\widetilde{\mu}^{2}$ (since $\widetilde{F}^{\prime}$ is an increasing function the average value of $\cos \Phi$ is positive). The correlation will then be larger at $0^{\circ}$ than at $180^{\circ} *$.

The physical interpretation is very clear: when the clusters are longitudinal, energy-momentum conservation in the cluster decay tends to favor particles going into opposite directions. However when the cluster transverse motion is large, both particles tend to follow its motion, and this effect finally wins over the first one.

\subsection{A specific model for cluster decay}

It seems difficult to go further without making some specific assumptions about cluster production and decay. Although these assumptions may turn out not to be

\footnotetext{
* A Monte-Carlo calculation by Berger and Ellis give the same result. We thank E. Berger for showing us the $\varphi$-dependence of the correlation in his calculation.
} 
entirely correct, we believe that they provide a useful illustration of the general formalism and may give hints on ways to organize the experimental data.

Our first assumption concerns cluster decay. We assume that the function $F\left(m^{2} ; M^{2}\right)$ is an exponential in $m^{2}$

$$
F\left(m^{2} ; M^{2}\right) \propto \exp \left(\frac{\lambda}{2 M} m^{2}\right)
$$

in order to obtain an exponential form for the particle distribution in the cluster rest frame:

$$
F=\frac{\gamma}{4 \pi} \exp (-\lambda E)
$$

Since $m^{2}=M^{2}+\mu^{2}-2 M E$. The normalization constant $\gamma$ in (17) is calculated in appendix A. The behavior (16) or (17) can be obtained approximately by a suitable choice of the coefficients $g_{n}$ in $(7)$ : for example in the approximation that the pions are massless we have $[11]$ :

$$
\Gamma_{n}\left(K^{2}\right)=\frac{2 \pi^{n-1}}{(n-1) !(n-2) !}\left(K^{2}\right)^{n-2},
$$

and choosing $g_{n}=(\lambda / 2 \pi M)^{n-2}(n-2) ! / n$ gives the behavior (16). For the two-particle distribution $\widetilde{F}$ we also choose an exponential in $m^{2}$. Although this choice does not satisfy exactly the energy momentum sum rules, we believe that it displays the essential features that we need $*$.

With the choice (17) we can perform the $\Phi$ and $Y$ integrations in (11), provided we forget about the limits of integration due to the positivity of $\mathrm{m}^{2}$. Since the decay is exponential, this is an excellent approximation except for large values of $p_{\perp}$. We then get

$$
\rho(p)=\gamma \int \mathrm{d} M \mathrm{~d} K_{\perp} K_{\perp} f\left(K_{\perp}, M\right) K_{0}\left(\frac{\lambda \mu_{\perp} M_{\perp}}{M}\right) I_{0}\left(\frac{\lambda p_{\perp} K_{\perp}}{M}\right),
$$

where $I_{0}$ and $K_{0}$ are modified Bessel functions. The calculation of the two-particle correlation is identical and leads to the result:

$$
\widetilde{C}\left(p_{1}, p_{2}\right)=\gamma \int \mathrm{d} M \mathrm{~d} K_{\perp} K_{\perp} f\left(K_{\perp}, M\right) \mathrm{e}^{\lambda \tilde{\mu}^{2} / 2 M} K_{0}\left(\frac{\lambda \mu_{1} M_{\perp}}{M}\right) I_{0}\left(\frac{\lambda \widetilde{p}_{\perp} K_{\perp}}{M}\right) .
$$

Since $\tilde{p}_{\perp}=\left(\tilde{\mu}_{\perp}^{2}-\widetilde{\mu}^{2}\right)^{\frac{1}{2}}$, we see again from (20) that $\widetilde{C}$ depends only on the variables $\widetilde{\mu}$ and $\widetilde{\mu}_{\perp}$. The term $\exp \left(\lambda \widetilde{\mu}^{2} / 2 M\right)$ expresses the way in which the two particles are correlated in the cluster rest frame.

We can make two remarks about (20):

(i) In the case of longitudinal clusters, we may expect an approximate factoriza-

* In the case of massless pions, the choice $F\left(m^{2}\right) \propto\left(6+6 \lambda^{\prime} m^{2}+\lambda^{\prime 2} m^{4}\right) \exp \left(\lambda^{\prime} m^{2}\right)$ with $\lambda^{\prime}=\lambda / 2 M$ satisfies exactly the energy momentum constraints. 
tion of $\widetilde{C}$ as a function of $\widetilde{\mu}$ and $\widetilde{\mu}_{\perp}$, since $I_{0}$ disappears from the integrand in (20).

(ii) In the general case we can take the derivative of $\widetilde{C}$ with respect to $\widetilde{\mu}^{2}$, as in (15). The integrand is then proportional to

$$
\left(1-\frac{K_{\perp} I_{1}}{\widetilde{p}_{\perp} I_{0}}\right) \text {. }
$$

Unless $K_{\perp}$ is very small, the ratio $I_{1} / I_{0}$ will be of the order of unity, and we expect that $\widetilde{C}$ becomes a decreasing function of $\widetilde{\mu}^{2}$ as soon as $\left\langle K_{\perp}^{2}\right\rangle$ is significantly larger than $\left\langle p_{\perp}^{2}\right\rangle$.

To proceed further, we choose a reasonable form of the function $f\left(K_{1}, M\right)$ in order to be able to perform the integrations analytically. Despite the arbitrariness of our choice, we believe that some aspects of the final results will show general validity. With this hope in mind we do the following. We write $f\left(K_{\perp}, M\right)$ in the form

$$
f\left(K_{\perp}, M\right)=\frac{h(M)}{M^{2}} g\left(\frac{K_{\perp}}{M}\right),
$$

and choose for $g$ the following expression:

$$
g(x)=\delta \frac{e^{-A \sqrt{1+x^{2}}}}{\left(1+x^{2}\right)^{\frac{1}{4}}}
$$

with $x=K_{\perp} / M$. The normalization constant $\delta$ is given in appendix $\mathrm{A}$. With the choice (21) for the cluster distribution the average cluster transverse momentum squared is

$$
\left\langle K_{\perp}^{2}\right\rangle=\left\langle M^{2}\right\rangle\left(\frac{\Gamma\left(\frac{7}{2}, A\right)}{A^{2} \Gamma\left(\frac{3}{2}, A\right)}-1\right),
$$

where $\Gamma(a, b)$ is the incomplete gamma function. We remark that the argument of the $K_{0}$ function in (19) is always larger than one (since $\lambda \gtrsim 6$ ) and we can replace $K_{0}$ by its asymptotic approximation with an error of a few percent. Then, if there is no unfortunate misprint in eq. (8.6.22) of Erdelyi et al. [12] we have:

$$
\rho(p)=\sqrt{\frac{\pi}{2}} \frac{\gamma \delta}{\sqrt{\lambda m_{\perp}}} \frac{e^{-\sqrt{A^{2}+\lambda^{2} \mu^{2}+2 A \lambda \mu_{\perp}}}}{\sqrt{A^{2}+\lambda^{2} \mu^{2}+2 A \lambda \mu_{\perp}}} .
$$

The two-particle correlation is given by a formula analogous to (23), multiplied by $\exp \left(\lambda \tilde{\mu}^{2} / 2 M\right)$.

In order to show that (23) gives a reasonable one-particle distribution we have drawn $\rho(p)$ on fig. 1 for two typical values of the parameters:

(i) typically small value of $\left\langle K_{\perp}^{2}\right\rangle$ : we choose $A=25, \lambda=6(\mathrm{GeV})^{-1}$ so that

$$
\left\langle K_{1}^{2}\right\rangle=0.085\left\langle M^{2}\right\rangle \text {. }
$$

If we assume a sharp $M$ distribution, the energy sum rule fixes $\langle M\rangle$ and $\left\langle M^{2}\right\rangle$ : we find $\left\langle M^{2}\right\rangle \sim 3 \mathrm{GeV}^{2}$ and

$$
\left\langle K_{1}^{2}\right\rangle \simeq 0.25 \mathrm{GeV}^{2} \text {. }
$$




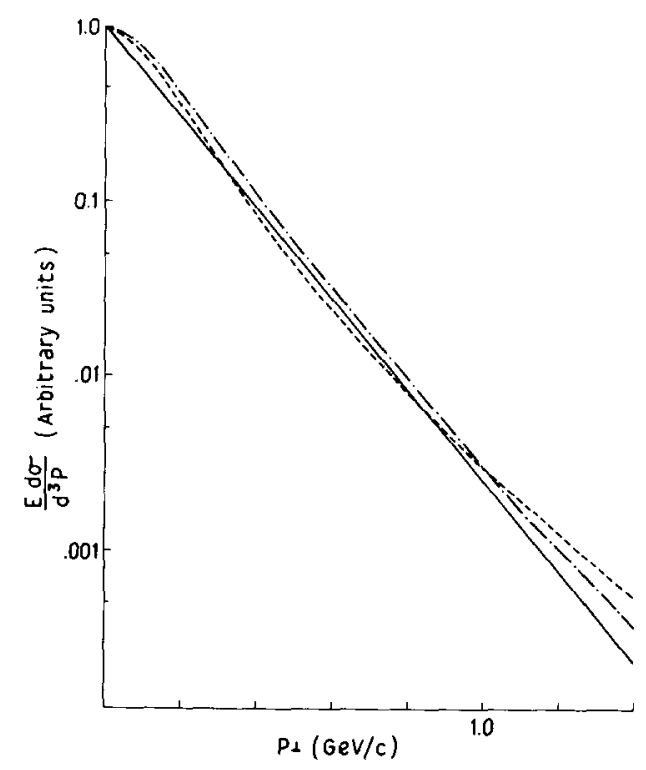

Fig. $1 . E \mathrm{~d} \sigma / \mathrm{d}^{3} p$ as a function of $p_{\perp}$. Dashed line: $\left\langle K^{2}\right\rangle=1.2 \mathrm{GeV}^{2}[A=4, \lambda=9$ in eq. (21) $]$. Dotted, dashed line: $\left\langle K_{\perp}^{2}\right\rangle=0.25 \mathrm{GeV}^{2}[A=25, \lambda=6]$. The curve exp $\left(-6 p_{\perp}\right)$ is given for comparison (full line).

(ii) typically large value of $\left\langle K_{1}^{2}\right\rangle$ : we choose $A=4, \lambda=9 \mathrm{GeV}^{-1}$ so that:

$$
\left\langle K_{\perp}^{2}\right\rangle=0.70\left\langle M^{2}\right\rangle \simeq 1.2 \mathrm{GeV}^{2},
$$

since we have $\left\langle M^{2}\right\rangle \sim 1.7 \mathrm{GeV}^{2}$. In this latter case it is interesting to notice the deviation of $\rho(p)$ from an exponential when $p_{\perp} \sim 1 \mathrm{GeV}$. This deviation results from a completely different mechanism from that of Pokorski and Van Hove [6] who attribute it to large mass clusters. Here we obtain it as a consequence of the large transverse momentum of the clusters.

It is interesting to compute the correlation at $y_{1}=y_{2}=0$ in both cases as a function of the azimuthal angle $\varphi$. If we fix $p_{1 \perp}$ and $p_{2 \perp}$ at their average value we immediately obtain from (23) the asymmetry:

$$
A_{s}=\frac{\widetilde{C}\left(\varphi=0^{\circ}\right)-\widetilde{C}\left(\varphi=180^{\circ}\right)}{\widetilde{C}\left(\varphi=0^{\circ}\right)+\widetilde{C}\left(\varphi=180^{\circ}\right)} ;
$$

we find

$$
A_{s}=-0.25
$$

in the first case (small $\left.\left\langle K_{\perp}^{2}\right\rangle\right)$ and

$$
A_{s}=+0.29
$$


in the second case $\left(\operatorname{large}\left\langle K_{\perp}^{2}\right\rangle\right)$ in agreement with the qualitative discussion given previously.

\subsection{Remarks on cluster correlations}

We conclude this section by some comments on the complications which arise when the clusters are not independent. Then the correlation defined as usual by

$$
C\left(p_{1}, p_{2}\right)=\frac{1}{\sigma_{\text {in }} \mathrm{d}^{3} p_{1} \mathrm{~d}^{3} p_{2}}-\frac{E_{1} E_{2} \mathrm{~d} \sigma}{\sigma_{\text {in }}}\left(\frac{E_{1} \mathrm{~d} \sigma}{\mathrm{d}^{3} p_{1}}\right) \frac{1}{\sigma_{\text {in }}}\left(\frac{E_{2} \mathrm{~d} \sigma}{\mathrm{d}^{3} p_{2}}\right)
$$

is not equal to $\widetilde{C}\left(p_{1}, p_{2}\right)$, since particles coming from different clusters are now coirelated. There are (at least) two reasons why we expect some correlations between clusters: (i) existence of long range rapidity correlations due, for example, to a twocomponent mechanism for cluster production and (ii) existence of azimuthal correlations between clusters because of transverse momentum conservation.

Both effects are difficult to estimate, because they turn out to be strongly model dependent. Let us first examine the influence of long range rapidity correlations. A first step in order to minimize their influence would be to use as normalization in (24) the non-diffractive cross section $\sigma_{\mathrm{ND}}$ instead of the total inelastic cross section $\sigma_{\text {in }}$, or to use a specific model for diffraction $[3,5]$ in order to calcualte explicitly the long range part of the correlations. Either procedure probably allows to determine the short range component within a $10-15 \%$ error.

The problem of azimuthal correlations between clusters is serious only if the clusters have a large $\left\langle K_{1}^{2}\right\rangle$. Assuming an IEM of cluster production we have a twocluster inclusive cross section of the form [13]:

$$
\frac{1}{\sigma} \frac{K_{10} K_{20} \mathrm{~d} \sigma}{\mathrm{d}^{3} K_{1} \mathrm{~d}^{3} K_{2}} \cong f\left(K_{1 \perp}\right) f\left(K_{2,1}\right)\left(1-\alpha K_{1 \perp} K_{2 \perp} \cos \theta\right),
$$

where $\theta$ is the azimuthal angle between the clusters and $\alpha \simeq 2 /\left(\left\langle n_{c l}\right\rangle\left\langle K_{\perp}^{2}\right\rangle\right)$. If the production mechanism is of the MPM type, $\alpha$ will be a decreasing function of the rapidity difference between the clusters, which means that the azimuthal correlation at zero rapidity difference is larger than in (25), since the transverse momentum sum rule [14] must be satisfied in either case. Hence we see that azimuthal correlations between clusters are model-dependent. In order to get an order of magnitude of the effect, we nevertheless assume (25), since we have no other reliable formula in the MPM case.

It is shown in appendix B that the correlation takes the following form:

$$
\begin{aligned}
& C\left(p_{1}, p_{2}\right)=\rho\left(p_{1 \perp}\right) \rho\left(p_{2 \perp}\right)\left\{-\alpha p_{1 \perp} p_{2 \perp} \hat{\rho}\left(p_{1 \perp}\right) \hat{\rho}\left(p_{2 \perp}\right) \cos \varphi\right. \\
& \left.\quad+\widetilde{C}\left(p_{1}, p_{2}\right) / \rho\left(p_{1 \perp}\right) \rho\left(p_{2 \perp}\right)\right\} .
\end{aligned}
$$

The coefficient of $\cos \varphi$ in (26) is typically of the order of $20 \%$ when $\left\langle K_{1}^{2}\right\rangle$ is large and $p_{1 \perp}, p_{2 \perp}$ are close to their average values. We can therefore hope that two- 
particle correlations are not significantly affected by azimuthal cluster correlations.

\section{Summary and conclusions}

We here summarize the two main points of our paper which we believe should be of interest to experimentalists:

(i) If the proper amount of the product of single-particle inclusive cross section has been removed from the two-particle cross section, the remaining correlation function should depend mostly on $\tilde{\mu}$ and $\widetilde{\mu}$ defined in eq. (14). This is important because the two particle inclusive cross sections depend generally on four variables in the central region (for example, $\widetilde{\mu}, \widetilde{\mu}_{1}, p_{1 \perp}, p_{2 \perp}$ ) and not just on two. Therefore, to test whether the short range part of the two-particle correlation is mainly determined by the phase space of cluster decay, one should organize the data in terms of different variables in order to find out whether the variation of the two-particle correlation is for the most part dependent on the two variables $\widetilde{\mu}$ and $\widetilde{\mu}_{\perp}$ only.

(ii) If there is transverse motion of clusters, this would indeed be an important indication that cluster formation is an essential ingredient in high energy multiparticle reactions. If it turns out that the variation with $\widetilde{\mu}$ and $\widetilde{\mu}_{\perp}$ accounts for the major part of the short range two particle correlation then the following test for cluster transverse momentum can be performed: hold $\widetilde{\mu}_{\perp}$ fixed and vary $\widetilde{\mu}_{\text {. Then the correlation }}$ function is predicted to rise if $\left\langle K_{\perp}\right\rangle$ is small and to fail if $\left\langle K_{\perp}\right\rangle$ is large. Another test is provided by the dependence of the correlation function on the azimuthal angle $\varphi$ at $y_{1}=y_{2}=0$ : for $\left\langle K_{1}\right\rangle$ small the correlation is larger at $\varphi=180^{\circ}$ than at $\varphi=0^{\circ}$, while for $\left\langle K_{1}\right\rangle$ large the opposite is expected.

Though this work is a rather crude attempt towards the understanding of transverse momentum correlations, we hope that it will provide the impetus for looking at the data in more imaginative ways.

\section{Appendix A. Normalizations}

In this appendix we give for completeness the normalization constants $\gamma$ and $\delta$ which appear in the one-particle distribution (17) and in the cluster distribution (21).

If the one-particle distribution within a cluster is given by (17) we have for the normalization constant

$$
\gamma=\frac{\langle n\rangle \lambda \mu}{\mu^{2} K_{1}(\lambda \mu)},
$$

so that in the cluster rest frame:

$$
\int \frac{E}{\sigma} \frac{\mathrm{d} \sigma}{\mathrm{d}^{3}} \frac{\mathrm{d}^{3} q}{E}=\langle n\rangle .
$$


The average particle energy $\langle E\rangle$ in the cluster rest frame is

$$
\langle E\rangle=\frac{1}{\lambda}\left(2+\lambda \mu K_{0}(\lambda \mu) / K_{1}(\lambda \mu)\right)
$$

and from the energy sum rule we have $\langle M\rangle=\langle n\rangle\langle E\rangle$. The average transverse momentum squared $\left\langle q_{\perp}^{2}\right\rangle$ which is needed in (2) is given by

$$
\left\langle q_{\perp}^{2}\right\rangle=\frac{2}{3}\left\langle E^{2}-\mu^{2}\right\rangle=\frac{2}{\lambda}\langle E\rangle .
$$

In order to compute the constant $\delta$ in (21) we evaluate the integral

$$
\begin{aligned}
\int f\left(K_{1}, M\right) \mathrm{d}^{2} K_{1} \mathrm{~d} M \mathrm{~d} Y & =2 \pi \delta \int h(M) \mathrm{d} M \mathrm{~d} Y \int x \mathrm{~d} x \frac{\mathrm{e}^{-A \sqrt{1+x^{2}}}}{\left(1+x^{2}\right)^{\frac{1}{4}}} \\
& =2 \pi \delta A^{-\frac{3}{2}} \Gamma\left(\frac{3}{2}, A\right) \int h(M) \mathrm{d} M \mathrm{~d} Y,
\end{aligned}
$$

where $\Gamma(a, b)$ is the incomplete gamma function. Thus:

$$
\delta=A^{\frac{3}{2}}\left\langle n_{\mathrm{cl}}\right\rangle\left[2 \pi \Gamma\left(\frac{3}{2}, A\right) \int h(M) \mathrm{d} M \mathrm{~d} Y\right]^{-1},
$$

so that

$$
\int f\left(K_{\perp}, M\right) \mathrm{d}^{2} K_{\perp} \mathrm{d} M \mathrm{~d} Y=\left\langle n_{\mathrm{cl}}\right\rangle .
$$

The average value $\left\langle M^{2}\right\rangle$ used in $(22)$ is to be defined with respect to $h(M)$ :

$$
\left\langle M^{2}\right\rangle=\left(\int h(M) M^{2} \mathrm{~d} M\right)\left(\int h(M) \mathrm{d} M\right)^{-1} .
$$

\section{Appendix B. Transverse correlations between clusters}

We assume that the two-cluster inclusive cross section is given by (25) and that the one-particle distribution within a cluster is exponential (17). A straightforward calculation leads to the following result:

$$
p_{\perp} \rho\left(p_{\perp}\right) \hat{\rho}\left(p_{\perp}\right)=\gamma \int \mathrm{d} K_{\perp} K_{\perp}^{2} K_{0}\left(\frac{\lambda M_{\perp} \mu_{\perp}}{M}\right) I_{1}\left(\frac{\lambda K_{\perp} p_{\perp}}{M}\right),
$$

where $\rho$ is defined in eq. (26) [compare with (19)]. In order to simplify the notations, we have neglected the dependence upon the cluster mass. In order to estimate $\hat{\rho}$ we equate $K_{\perp}$ to its average value in (B.1) and (19), which should be valid if $p_{\perp} \sim\left\langle p_{1}\right\rangle$. We then find

$$
p_{\perp} \hat{\rho}\left(p_{\perp}\right)=\frac{\left\langle K_{\perp}\right\rangle I_{1}\left(\lambda\left\langle K_{\perp}\right\rangle p_{\perp} / M\right)}{I_{0}\left(\lambda\left\langle K_{\perp}\right\rangle p_{\perp} / M\right)}
$$

For $\left\langle K_{\perp}\right\rangle \sim 1 \mathrm{GeV}$ we find that $p_{\perp} \hat{\rho}\left(p_{\perp}\right)$ is of the order of $\sim 0.7 \mathrm{GeV}$, which means that the coefficient of $\cos \varphi$ in $(26)$ is $\sim 20 \%$ at ISR where $\left\langle n_{\mathrm{cl}}\right\rangle \sim 4$. We can make a 
more complete calculation by using the form (21) of $f\left(K_{1}\right)$. We find

$$
\hat{\rho}\left(p_{\perp}\right)=\frac{\lambda M}{\left(A+\lambda m_{\perp}\right)^{2}+\lambda^{2} p_{\perp}^{2}} \quad\left(1+\sqrt{\left(A+\lambda m_{\perp}\right)^{2}+\lambda^{2} p_{\perp}^{2}}\right) .
$$

With the values $A=4, \lambda=6 \mathrm{GeV}^{-1}$ and $p_{\perp} \sim 0.3 \mathrm{GeV}$ we find again that $p_{\perp} \hat{\rho}\left(p_{\perp}\right)$ is $\sim 0.7$, in agreement with the previous estimate.

\section{References}

[1] P. Pirila and S. Pokorski, Phys. Letters 43B (1973) 502.

[2] F. Hayot and A. Morel, Nucl. Phys. B68 (1974) 323.

[3] E. Berger and G. Fox, Phys. Letters 47B (1973) 162.

[4] W. Schmidt - Parzefall, Phys. Letters 46B (1973) 399.

[5] M. LeBellac, H. Miettinen and R.G. Roberts, Phys. Letters 48B (1974) 115.

[6] S. Pokorski and L. Van Hove, CERN preprint TH1772 (1973).

[7] F.S. Henyey, Phys. Letters 45B (1973) 363, 469.

[8] R. Hwa, Phys. Rev. D8 (1973) 1131;

C. Hamer and R. Peierls, Phys. Rev. D8 (1973) 1358.

[9] F.S. Henyey, University of Michigan preprint UM74-8.

[10] L. Caneschi, Private communication.

[11] F. Lurçat and P. Mazur, Nuovo Cimento 31 (1964) 140.

[12] A. Erdelyi et al., Tables of integral transforms, vol. II, (McGraw Hill, 1954).

[13] L. Caneschi, Proc. of the 7th Moriond Rencontre, ed. Tran Than Van, 1972.

[14] L.S. Brown, Phys. Rev. D5 (1972) 748. 